九州大学学術情報リポジトリ

Kyushu University Institutional Repository

\title{
Determination of Intramuscular Fat Content in Beef using Magnetic Resonance Imaging
}

\section{Lee, Sangdae}

Convergence components \& agricultural machinery application center, Korea Institute of Industrial Technology

Lohumi, Santosh

Department of Biosystems Machinery Engineering, Chungnam National University

Lim, Hyoun-Sub

Department of Applied Biology, Chungnam National University

Gotoh, Takafumi

Kuju Agricultural Research Center, Faculty of Agriculture, Kyushu University

他

https://doi.org/10.5109/1526313

出版情報: 九州大学大学院農学研究院紀要. 60 (1)，pp.157-162，2015-02-27. Faculty of Agriculture, Kyushu University

バージョン：

権利関係 : 


\title{
Determination of Intramuscular Fat Content in Beef using Magnetic Resonance Imaging
}

\section{Sangdae LEE ${ }^{1}$, Santosh LOHUMI', Hyoun-Sub LIM ${ }^{3}$, Takafumi GOTOH ${ }^{4}$, Byoung-Kwan $\mathrm{CHO}^{2 *}$ and Samooel $\mathrm{JUNG}^{5}$}

\author{
Kuju Agricultural Research Center, Kyushu University, \\ Kuju 4045-4, Taketa City, 878-020, Oita, Japan \\ (Received October 31, 2014 and accepted November 14, 2014)
}

\begin{abstract}
The amount and distribution of intramuscular fat in beef and pork are some of the most important quality characteristics because they highly influence the nutritional value and eating quality of the meat. Magnetic resonance imaging (MRI) is a suitable non-invasive alternative method that can provide both qualitative and quantitative information on beef meats with high spatial information. In this study, we used MRI technique in combination with image processing tools for the visualization and prediction of intramuscular fat distribution in beef meats. The results from image analysis were compared with the chemical reference result for the percentage of intramuscular fat. The results show a strong correlation $\left(R^{2}=0.98\right)$ between the MRI detected and chemically measured values. The distribution uniformity of intramuscular fat within the beef meats was evaluated by calculating their distribution and pixel size in the MRI according to four levels of beef quality grade $(1+, 1,2$, and 3$)$.
\end{abstract}

Key words: beef, magnetic resonance imaging, image processing, threshold, intramuscular fat

\section{INTRODUCTION}

The quality grade of beef carcass is estimated using several factors such as marbling score, meat color, fat color, firmness and texture of lean meat, and physiological maturity of the exposed longissimus dorsi muscle at the $12^{\text {th }} / 13^{\text {th }}$ rib-eye interface. The marbling score, which is the amount and distribution of intramuscular fat, is one of the most dominating determinants in Korean, Japan, and United States Department of Agriculture (USDA) quality grading. The marbling score is a component of the USDA beef quality grading system (beef marbling standard (BMS) in Korea and Japan), and refers to visible fat found between muscle fiber bundles within the rib-eye. Intramuscular fat also quantifies the fat found between muscle fiber bundles within the rib-eye and determined by chemical extraction of lipid from a thin facing of the exposed rib-eye. Therefore, the percentage intramuscular fat is an objective measurement that quantifies the total fat content within the rib-eye. There is a strong correlation between marbling score and intramuscular fat (Hassen et al., 1998). The marbling score

\footnotetext{
${ }^{1}$ Convergence components \& agricultural machinery application center, Korea Institute of Industrial Technology, 119, Jipyeongseonsandan 3-gil, Baeksan-myeon, Gimje-si, Jeollabuk-do, 576-881, South Korea

2 Department of Biosystems Machinery Engineering, Chungnam National University, 220 Gung-dong, Yuseong-gu, Daejeon, 305-764, South Korea

${ }^{3}$ Department of Applied Biology, Chungnam National University, 220 Gung-dong, Yuseong-gu, Daejeon, 305-764, South Korea

${ }^{4}$ Kuju Agricultural Research Center, Faculty of Agriculture, Kyushu University, Kuju, Oita, Japan

${ }^{5}$ Department of Animal Science and Biotechnology, Chungnam National University, 220 Gung-dong, Yuseong-gu, Daejeon, 305-764, South Korea

* Corresponding Author (E-mail: chobk@cnu.ac.kr)
}

of beef carcass is related to differences in eating quality. Beef cuts with high marbling score are more likely to be tender, juicy, palatable, and flavorful (Smith et al., 1987). Especially, consumers consider the marbling factor significant purchase decision factor in Korea and Japan. Since consumers have an extraordinary preference for highly marbled meats, beef grading has been made based on the marbling score.

The visual inspection of field graders incorporates several variables including amount, size, and distribution of intramuscular fat within the exposed rib-eye, as well as lean and fat color. Machine vision technology for the measurement of the $12^{\text {th }} / 13^{\text {th }}$ rib-eye interface, such as $\mathrm{X}$-ray computed tomography (CT) and MRI of the entire cattle carcass, studied as typical non-invasive techniques for predicting the marbling score of beef carcass (Collewet et al., 2005). At first, researchers utilized digital image processing indicating the amount of marbling and other visible attributes of rib-eye to predict the marbling score with high accuracy. Shackelford et al. (2003) used a specially developed image analysis system for on-line prediction of the yield grade, longissimus dorsi muscle area, and marbling score of cattle carcasses. Prediction equations accounted for $90,88,90,88$, and $76 \%$ of the variation in the calculated yield grade, longissimus dorsi muscle area, preliminary yield grade, adjusted preliminary yield grade, and marbling score, respectively. Wyle et al. (2003) tested the prototype BeefCam video imaging system for classifying beef carcasses into palatability groups. Images of longissimus doris muscles were captured and were analyzed using Model I and II. Moore (2010) used the video image analysis-computer vision system (VIA-CVS) to develop and outline an appropriate method to accurately calculate the marbling score at commercial processing facility production speeds. Furthermore, it was reported that VIS-CVS exhibited 
greater accuracy and precision than any other instrument used to predict the marbling score.

MRI is based on the magnetic resonant properties of protons associated with water and lipid molecules of the tissues and results in a range of signal intensities capable of distinguishing numerous tissues and organs, including fat and muscle. A series of consecutive images can be reconstructed to render a volume measurement of the special region of interest. In carcass composition analysis using MRI, early studies demonstrated close association between physical (or chemical) dissection and MRI prediction on the muscle and fat contents of specific regions. Mitchell et al. (2001) used MRI to measure the entire pig and to compare the MRI volumes of fat, muscle, heart, liver, and brain with the weights obtained by dissection. Collewet et al. (2005) used a 1.5T MRI system to calculate the lean meat percentage of pig carcasses and automatic image segmentation was performed to quantify the volume of lean meat in the pig carcass images. Monziols et al. (2006) measured the tissue composition of pig carcasses and cuts using a low field MRI imager. They reported that MRI provided good predictions of muscle, total fat, and subcutaneous fat contents with $\mathrm{R}^{2}$ ranging from 0.951 to 0.997 , but prediction results of intramuscular fat contents yielded low coefficients of determination $\left(\mathrm{R}^{2}=0.093 \sim 0.522\right)$ with the exception of belly $\left(\mathrm{R}^{2}=0.798 \sim 0.837\right)$. In another study, Monziols et al. (2005) reported that segmentation of pure tissue and partial volume voxels allows the separation of muscle and fat tissue including the find insertions of intramuscular fat.

The quality aspect of muscle food is determined by the amount and distribution of intramuscular fat and the muscle water properties. Not only the quantity of intramuscular fat, but also the location of intramuscular fat is an important consideration in determining meat quality grade. This study investigated the potential of the MRI technique to determine the amount and distribution of intramuscular fat in beef meats. Our hypothesis was based on the intensity difference between fat and lean meat content in the proton spin-lattice relaxation (T1) weighted MRI image, which produces distinct results upon image processing. The object of this study is to investigate the feasibility of the future use of on-line MRI system for nondestructive measurement of inside quality of meat products based on their sensory qualities such as marbling, tenderness and juiciness.

\section{MATERIALS AND METHODS}

\section{BEEF MEAT}

The aim of this study was to determine the amount and distribution of intramuscular fat in beef meats. Four levels of beef meats (i.e., 1+, 1, 2, 3 grade ribeyes) based on the beef quality grade were purchased from a local market to achieve different ranges of intramuscular fat values. Thus, the obtained beef meats were different in thickness and size. Vacuum-packaged beef meats were stored in a portable ice box before MRI analysis. Fig. 1 shows $1+$ and 2 grade beef meats for MRI test.

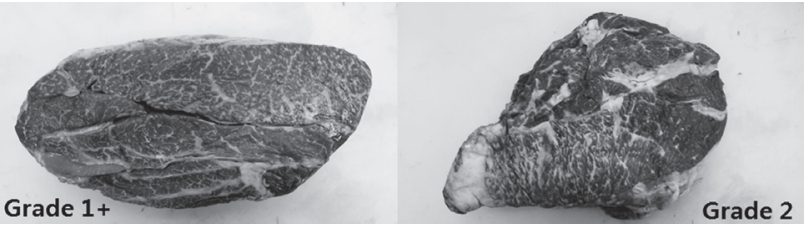

Fig. 1. $1+$ and 2 grade beef meats for MRI test.

\section{MRI IMAGE ACQUISITION}

MRI scans of beef meats were taken using the medical equipment Philips Achieva 3.0T MRI system (at the Korea Basic Science Institute, Ochang, Korea) equipped with a head coil. Prior to MRI experiment care had to be taken for the choice of the image acquisition protocol because these parameters highly influence the signal-tonoise ratio which affects tissue contrast. In this study, the MRI volume data set of $5 \mathrm{~mm}$ thick slices was obtained from a sequence of T1 images (with $10 \mathrm{~ms} \mathrm{TE}$, $700 \mathrm{~ms}$ TR, and $90^{\circ}$ flip angle) at different fields of view for different grade beef meats. The parameters were chosen to optimize the image contrast by compromising between resolution and signal-to-noise ratio. The T1 data are highly correlated with the chemical analysis of fat content because fat has large longitudinal and transverse magnetization, and appear bright on a T1 weighted image (Kullberg et al., 2009). The obtained image resolution was $3,216 \times 2,136$ pixels and 16 -bits. Fig. 2 shows the MRI images of beef meats with four grades, in which the fat is associated with the whiter region.

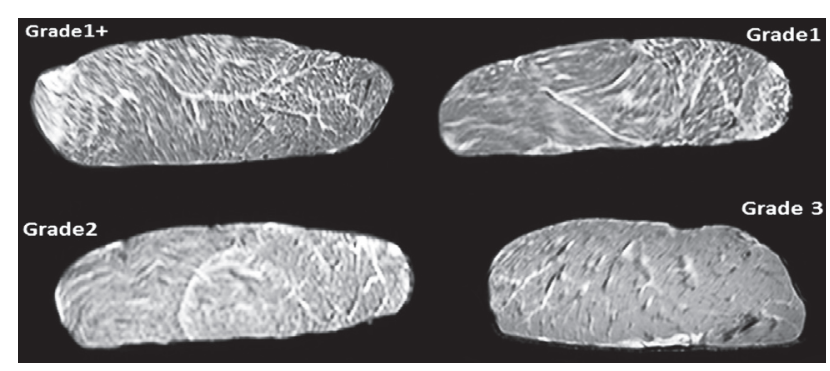

Fig. 2. MRI images of different grades of beef samples. Pixels corresponding to fat are in white.

\section{CHEMICAL ANALYSIS}

Intramuscular fat content of beef meats was determined using the Soxhlet extraction method (AOAC Method 960.39). First, the meat was minced and mixed well. A defined and weighted meat was transferred into an aluminum vessel and mixed with sea sand and ethanol. Meats were dried for $1 \mathrm{~h}$ at $105^{\circ} \mathrm{C}$ in a drying oven. Then, meats were cooled to room temperature in a desiccator and weighted. The dried meat was transferred into the Soxhlet equipment. Extraction was achieved with petroleum ether for $6 \mathrm{~h}$ at $60^{\circ} \mathrm{C}$. After the solvent was evaporated, the flask containing the fat was dried for $1 \mathrm{~h}$ at $100^{\circ} \mathrm{C}$ and cooled to room temperature in a desiccator. Finally, intramuscular fat content was calculated as follows.

Intramuscular fat $(\%)=\frac{\text { Weight of intramuscular fat }}{\text { Weight of beef meat }} \times 100$ 


\section{MRI ANALYSIS}

MRI was processed using the ImageJ software. The ImageJ image analysis software is a Java-based open source image enumeration software package publically available at the US National Institutes of Health website (NIH, Bethesda, MD, USA, http://rsb.info.nih.gov/ij/). Image $J$ is the global software which prove its reliability in term of image analysis in various fields. ImageJ is simple analysis software, easy to use, and provides a vast range of useful options to display, edit, analyze, process and save images.

In this study, we used ImageJ to characterize and predict the amount and distribution of intramuscular fat within beef meats using MRI. The total beef slice and intramuscular fat images were extracted from the original MRI and to calculate the total areas of the obtained beef slice and intramuscular fat. First, the total beef slice was extracted from the MRI (Fig. 3(a)). This step was performed using the threshold method based on histogram analysis techniques (Ballerini and Bocchi, 2006). The threshold values were determined based on the segmentation of the MRI. This was done using the threshold command, in which the grayscale values for the background pixels are higher than the values of the pixels representing total beef slice (Fig. 3(b)). The total area of the obtained beef slice image was measured using the analyze particles command (Fig. 3(d). Next, the intramuscular fat image was selected (Fig. 3(c)). The total number, total area, and area of each particle of the obtained intramuscular fat image were measured (Fig. 3(e)). Fig. 3 illustrates the procedure of the MRI analysis using ImageJ for the prediction of intramuscular fat content in beef meats.

The beef meat pixels were classified in two categories, intramuscular fat and muscle, depending on the signal intensity. The maximum of the signal intensity was around 2000. We computed the threshold values from 1100 to 1300 and chose the one that gave the best match with the result of the chemical analysis of beef meats. In this study, we used four grades of beef meats. The MRI image intensities slightly fluctuated within the different grades of beef meats, resulting in different threshold values, and this variation was within the range of 20 . However, the prediction of fat content in different beef meats belonging to the same grade was accurately achieved using a single fixed threshold value. The threshold values ranged from 1175 to 1195; however a fixed threshold value for each group was used.

Based on thresholding, each pixel was assigned to a particular category of intramuscular fat or muscle. If the intensity was above the threshold value, this pixel was classified as fat; on the contrary, if the intensity was below the threshold value, the pixel was classified as muscle. By following this procedure, after measuring the cross-sectional areas of beef slice and total pixel area of intramuscular fat, the intramuscular fat content was calculated using the densities of fat $(0.92 \mathrm{~g} / \mathrm{mL})$ and muscle $(1.0 \mathrm{~g} /$ $\mathrm{mL})$ as follows.

Intramuscular fat content (\%)

$$
\begin{aligned}
& =\frac{\text { Weight of intramuscular fat }}{\text { Weight of beef slice }} \times 100 \\
& =\frac{V_{F} O_{F}}{V_{M} \rho_{M}+V_{F} O_{F}} \times 100 \\
& =\frac{P_{F} H_{F} O_{F}}{V_{M} H_{M} O_{M}+P_{F} H_{F} O_{F}} \times 100
\end{aligned}
$$

where, $V_{M}$ is the volume of muscle, $V_{F}$ is the volume of intramuscular fat, $\rho_{M}$ is the density of muscle, $\rho_{F}$ is the density of intramuscular fat, $H_{M}$ is the thickness of muscle, $H_{F}$ is the thickness of intramuscular fat, $P_{M}$ is the total pixel number of muscle, and $P_{F}$ is the total pixel number of intramuscular fat.

Since the thickness of intramuscular fat and muscle are equal, $H_{M}$ and $H_{F}$ can be eliminated.

$$
\text { Intramuscular fat content }(\%)=\frac{P_{F} O_{F}}{P_{M} \rho_{M}+P_{F} O_{F}} \times 100
$$

\section{RESULTS AND DISCUSSION}

Table 1 summarizes the results of the dissection of beef meats of different grades. The fat content of the beef meats varied from $\sim 27 \%$ to $~ 8 \%$. After chemical analysis result for the fat content, the acquired MRI images were analyzed and the threshold value based on differ-

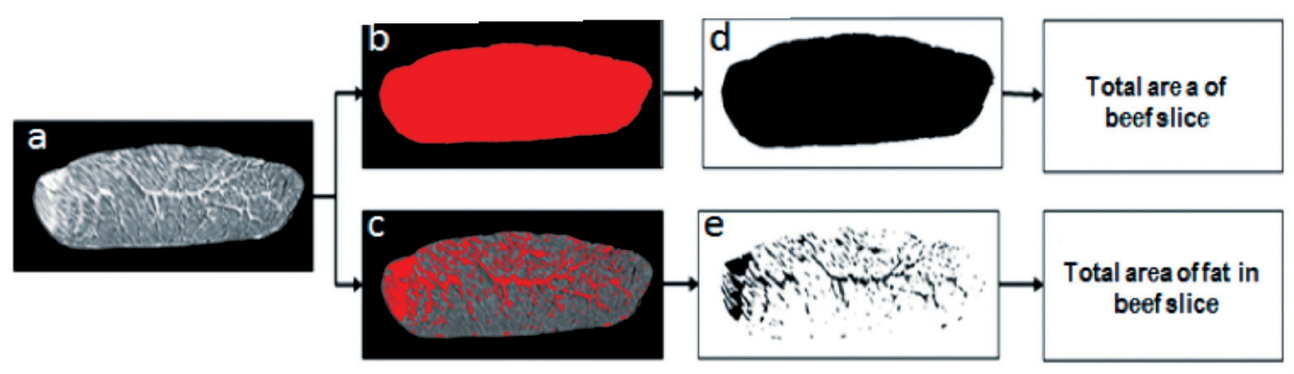

Fig. 3. Main steps involved in extraction of fat pixels from the MRI image and application of Image J image processing. (a) Original MRI image of beef meat, (b) Selected total beef slice image, (c) Selected fat region image, (d) Extracted total beef slice image from background, and (e) Extracted fat region image from muscle and background. 
ence between the signal intensities of the different tissues (intramuscular fat and muscle). The general concept of the thresholding method is that a detected value higher than the threshold is counted as intramuscular fat pixel, a value lower than the threshold is regarded as muscle pixel, and a value similar to the threshold is considered as uncertain, meaning that it may belongs to either group.

Nevertheless, if the fat content for different grade beef meats is detected on the basis of the MRI processing, the detection efficiency was more precise for high fat content samples than for low fat content. The overall variation between chemically analyzed and MRI identified fat percentage was very low. Several reasons may explain errors in fat detection using MRI analysis. The main reason of this error on the image analysis might be the fact that this method is based on the thresholding process, which means that one pixel is classified as pure fat or not. In fact, some pixels are located at the interface between muscle and fat may contain both tissues. This phenomenon is known as the partial volume effect. Since the slice thickness is equal to $5 \mathrm{~mm}$, this may affect an important number of pixels depending on the orientation of the slice.

In a comparison study (Yamaguchi et al., 1992), the extent and content of intramuscular fat (marbling) in $M$. longissimus thoracis were evaluated by MRI. The result showed that MRI is a reliable tool to evaluate the marbling and the associated meat quality on the carcass of beef cattle; however it may not be as effective in all breeds of cattle, especially those with smaller amount of intramuscular fat. The resultant images for the identification of fat content in the MRI corresponding to Fig. 2 are shown in Fig. 4, which shows the distribution of intramuscular fat. Fig. 5 shows the MRI of a $1+$ grade beef meat after processing. Fig. 6 shows the detected fat content in the MRI of different grade beef meats after applying the threshold value against the percentage of fat content measured with the previously described chemical method. The $\mathrm{R}^{2}$ of 0.986 indicates that there is a strong correlation between the MRI detected and chemically measured values. Six traits such as; brightness, dryness, hardness, juiciness, marbling degree and size of marbling are common parameters to define beef quality. The size of marbling is referred to the homogeneity in the fat distribution in the beef meat, with more homogeneity producing a better quality. Eikelenboom et al., (1996) reported that sensory tenderness, juiciness and flavor were moderately related to marbling. They sug- gested that distribution of intramuscular fat is related to the eating quality of meat.

By using the purposed MRI method in conjunction with image processing techniques, the distribution of intramuscular fat (size of marbling) can also be assessed. For example, marbling in Iberian ham has been studied using MRI (Antequera et al., 2003). Because of the fat voxel size and distribution, segmentation of the images could be applied. Therefore, texture analysis was chosen to quantify the heterogeneity of the intensity of the image due to the presence of very fine insertion of fat. Fig. 7 shows the histogram for the fat pixel size distribution. The fat pixel size in the MRI was calculated using the analyze particles command contained in the Image J software. Fig. 7 shows the total pixel area of intramuscular fat on the y-axis and the number of corresponding pixels on the $\mathrm{x}$-axis. It can be seen that intramuscular fat is more uniformly distributed in high-grade beef meats than in low-grade meat. Thus, this technique can be considered reliable for beef grading based on the marbling degree and size of marbling which are two important quality parameters of beef quality grading.

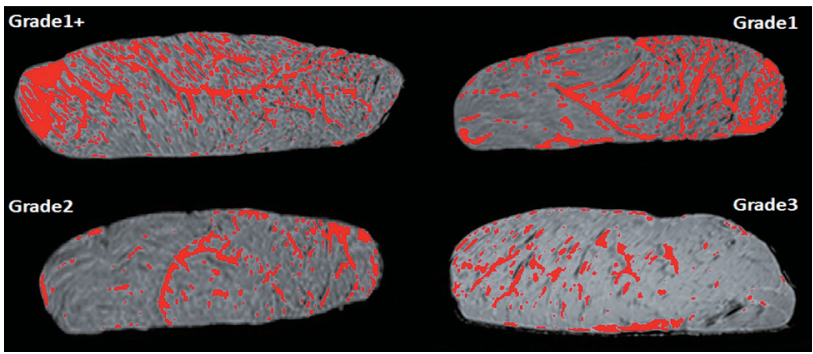

Fig. 4. MRI images of different grades of beef meats after image processing. Pixels corresponding to intramuscular fat are in red.

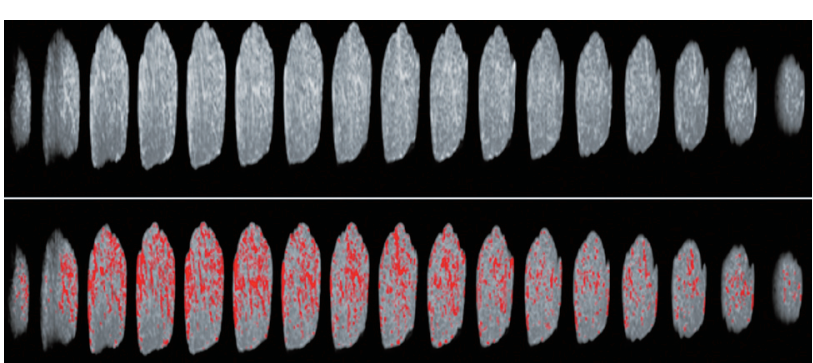

Fig. 5. MRI images from a $1+$ grade beef meat after MRI acquisition (upper row) and after image processing (lower row).

Table 1. Percentage of pixels in MRI image of beef meats identified as containing intramuscular fat after applying thresholding method.

\begin{tabular}{|c|c|c|c|c|c|}
\hline $\begin{array}{l}\text { Quality } \\
\text { Grade }\end{array}$ & No. of Samples & $\begin{array}{c}\text { Average Fat \% } \\
\text { (Chemical analysis) }\end{array}$ & $\begin{array}{c}\text { Average Fat \% } \\
\text { (Image processing) }\end{array}$ & Variation (\%) & $\begin{array}{c}\text { Threshold } \\
\text { values }\end{array}$ \\
\hline $1+$ & 12 & 27.31 & 27.06 & 0.9 & 1125 \\
\hline 1 & 12 & 21.21 & 21.36 & 0.6 & 1140 \\
\hline 2 & 10 & 16.83 & 16.69 & 0.8 & 1145 \\
\hline 3 & 9 & 8.39 & 8.81 & 5.0 & 1180 \\
\hline
\end{tabular}




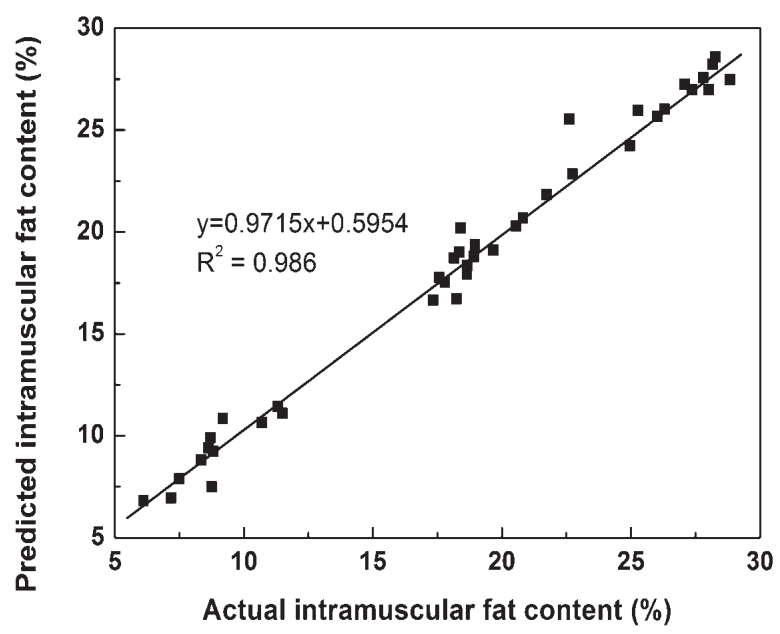

Fig. 6. Comparison of MRI estimated fat concentration against the reference chemistry.

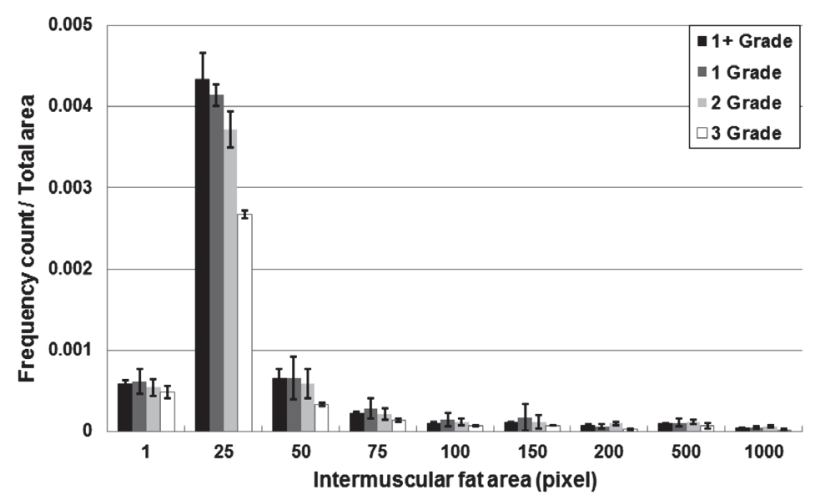

Fig. 7. Distribution analysis of fat particle size according to different grads of beef meats.

\section{CONCLUSION}

Beef marbling is regarded as the most important indicator of beef quality grade. Beef marbling is determined by the intramuscular fat content in the beef rib-eye region. Since the classification of the marbling score depend on the visual inspection of the graders, there is a slight difference between the marbling scores of different graders. Recently, machine vision and image processing technology have been considered as the most effective methods for automatic identification of the beef marbling score.

In this study, the MRI technique was used for the visualization and detection of intramuscular fat in beef meats. The Soxhlet extraction method (i.e., chemical method) was used to determine the intramuscular fat content. The ImageJ software and the threshold method were used for MRI analysis. The results from the MRI image analysis of beef meats revealed good performance and high detection efficiency. The high correlation $\left(\mathrm{R}^{2}=\right.$ 0.986) between MRI and chemical analysis methods for the determination of intramuscular fat content supports the finding that image analysis is a reliable tool for this kind of investigation. The excellent contrast between the intramuscular fat and muscle components of meat permits the use of MRI to quantify the intramuscular fat and muscle content in the intact carcass or various cuts of meat.

The MRI method can also measure the marbling size in beef meats which is an important quality factor and cannot be analyzed with the existing conventional or local analytical methods because of the presence of very fine insertion of intramuscular fat. Thus, the combination of the MRI technique and image processing tools provides accurate prediction of the fat content and marbling in beef meats and can be applied to various meat products. The potential of MRI technique for evaluating meat quality was proved. These results are encouraging for development and application of low-cost non-medical MRI system allowing automatic, nondestructive and precise evaluation of the quality of whole carcass in meat industry.

\section{ACKNOWLEDGEMENT}

This research was supported by Technology Commercialization Support Program, Ministry of Agriculture, Food and Rural Affairs (MAFRA), Republic of Korea.

\section{REFERENCES}

Antequera T., E. Muriel, P. G. Rodriguez, E. Cernadas and J. Ruiz. 2003 Magnetic resonance imaging as a predictive tool for sensory characteristics and intramuscular fat content of dry-cured loin. J. Sci. Food Agric. 83(4): 268 -274.

Ballerini L. and L. Bocchi. 2006 Genetic Programming for Prediction of Fat Content in Meat Images. Workshop on Evolutionary Computation (GSICE2). Siena, Italy, 15 September 2006.

Collewet G., P. Bogner, P. Allen, H. Busk, A. Dobrowolski, E. Olsen and A. Davenel. 2005 Determination of the lean meat percentage of pig carcasses using magnetic resonance imaging. Meat Sci. 70: 563-572.

Eikelenboom G., A. H. Hoving-Boling and P. G. V. D. Vender Wal. 1996 The eating quality of pork- The influence of intramuscular fat. Fleischwirtschaft. 3: 18-20.

Kullberg J., J. Brandberg, J. E. Angelhed, H. Frimmel, E. Bergelin, L. Strid and L. Lonn. 2009 Whole-body adipose tissue analysis: comparison of MRI, CT and dual energy X-ray absorptiometry. Br. J. Radiol. 82(974): 123-130.

Hassen A., M. M. Izquierdo, G. H. Rouse, D. E. Wilson and R. L. Willham. 1998 Prediction od marbling scores from percentage intramuscular fat. Animal Science Research Report. Iowa State University, Ames, IA, A. S. Leaflet R1413.

Mitchell A. D., A. M. Scholz, P. C. Wang and H. Song. 2001 Body composition analysis of the pig by magnetic resonance imaging. J. Anim. Sci. 79: 1800-1813.

Monziols M., G. Collewet, F. Mariette, M. Kouba and A. Davenel. 2005 Muscle and fat quantification in MRI gradient echo images using a partial volume detection method. Application to the characterization of pig belly tissue. Magn. Reson. Imaging 23: 745-755.

Monziols M., G. Collewet, M. Bonneau, F. Mariette, A. Davenel and M. Kouba. 2006 Quantification of muscle, subcutaneous fat and intermuscular fat in pig carcasses and cuts by magnetic resonance imaging. Meat Sci. 72: 146-154.

Moore C. B., P. D. Bass, M. D. Green, P. L. Chapman, M. E. O'Connor, L. D. Yates, J. A. Scanga, J. D. Tatum, G. C. Smith and K. E. 
Belk. 2010 Establishing an appropriate mode of comparison for measuring the performance of marbling score output from video image analysis beef carcass grading systems. J. Anim. Sci. 88: 2464-2475.

Shackelford S. D., T. L. Wheeler and M. Koohmaraie. 2003 On-line prediction of yield grade, longissimus muscle area, preliminary yield grade, adjusted preliminary yield grade, and marbling score using the MARC beef carcass imaging analysis system, $J$. Anim. Sci. 81(1): 150-155.

Smith G. C., J. W. Savell, H. R. Cross, Z. L. Carpenter, C. E. Murphey, G. W. Davis, H. C. Abraham, F. C. Parrish Jr. and B. W. Berry. 1987 Relationship of USDA quality grades to palatability of cooked beef. J. Food Qual. 10: 269-286.

Wyle A. M., D. J. Vote, D. L. Roeber, R. C. Cannell, K. E. Belk, J. A Scanga, M. Goldberg, J. D. Tatum and G. C. Smith. 2003 Effectiveness of the SmartMV prototype BeefCam system to sort beef carcasses into expected palatability groups. $J$. Anim. Sci. 81(2): 441-448.

Yamaguchi K., K. Ozutsumi, S. Ohwada, S. Yoneya, A. Suzuki, K. Matsumoto, K. Sakamoto, K. Yamaki, M. Yoshitake, K. Satoh and T. Hoshino. 1992 Improved evaluation by magnetic resonance imaging (MRimaging) on marbling in M. longissimus thoracis of carcasses of beef cattle. Anim. Sci. Technol. 63(9): 942-946. 